\title{
Fiber-laser-pumped, high-energy, mid-IR, picosecond optical parametric oscillator with a high-harmonic cavity
}

\author{
L. Xu", H.-Y. Chan, S-U. Alam, D.J. Richardson, and D.P. Shepherd \\ Optoelectronics Research Centre, University of Southampton, Southampton, SO17 1BJ, UK \\ *Corresponding author: l.xu@soton.ac.uk
}

Received Month X, XXXX; revised Month X, XXXX; accepted Month X, XXXX; posted Month X, XXXX (Doc. ID XXXXX); published Month X, XXXX

\begin{abstract}
We demonstrate the generation of high-energy, mid-IR, picosecond pulses in a high-harmonic-cavity optical parametric oscillator (OPO) that has a relatively compact cavity with a length that is a small fraction of that required to match the pump repetition rate. The $\mathrm{OPO}$, based on an $\mathrm{MgO}$-doped periodically poled $\mathrm{LiNbO}_{3}$ crystal, is pumped by a fiber master-oscillatorpower-amplifier system employing direct amplification and delivering $11 \mu \mathrm{J}, 150 \mathrm{ps}$ pulses at $1035 \mathrm{~nm}$. For a $1.554-\mathrm{m}$ long OPO cavity, resonating near-infrared signal pulses with a repetition rate that is the $193 \mathrm{rd}$ harmonic of the $1 \mathrm{MHz}$ pump are demonstrated. The mid-infrared idler output pulses, tunable from $2300 \mathrm{~nm}$ to $3500 \mathrm{~nm}$, are generated at a $1 \mathrm{MHz}$ repetition rate and have energies as high as $1.5 \mu \mathrm{J}$. (C) 2015 Optical Society of America

OCIS Codes: (190.4970) Parametric oscillators and amplifiers, (190.4400) Nonlinear optics, materials, (190.7110) Ultrafast nonlinear optics.
\end{abstract}

Tunable sources of short pulses in the mid-infrared (midIR) are useful for a number of applications, as this part of the spectrum corresponds to characteristic vibrational absorptions of organic materials [1,2]. In particular, short pulses with $\mu \mathrm{J}$-level energies in the mid-IR are interesting for organic material processing techniques such as resonant infrared pulsed laser deposition (RIR-PLD) [3,4]. Optical parametric generators (OPGs) are commonly used to generate high-energy pulses in the mid-IR, taking advantage of low-repetition rate, high-energy pump sources and high-nonlinearity crystals [5-7]. However, OPGs have a broad spectral output that limits their range of potential applications. Injection-seeded optical parametric amplifiers (OPAs) can effectively reduce the spectral bandwidth, but in order to generate widely tunable mid-IR pulses, tunable seed laser sources are required [8-10]. On the other hand, synchronously pumped optical parametric oscillators (OPOs) are attractive, due to their good efficiency and wide tunability, but pumping with the low repetition rate sources required for high pulse energies would normally demand very long cavity lengths to match the condition for synchronous pumping. Ultrashort pulses with energies as high as $0.65 \mu \mathrm{J}$ in the near- $\mathbb{R}$ and $0.19 \mu \mathrm{J}$ in the mid-IR have been reported from low-repetition $(<20 \mathrm{MHz})$ OPOs using either relay-imaging and cavity-dumping in a free-space resonator, or by employing an intracavity feedback optical fiber to take up most of the required cavity length [11-13].

In this letter we describe the use of a harmonic cavity as an alternative route to utilizing high-energy, lowrepetition rate pump sources whilst maintaining a compact overall design, as the cavity length is set to be a small but exact fraction of that required for true synchronous pumping. Previous work has concentrated on the use of such cavities to generate high-repetition-rate short pulses at the signal wavelength in femtosecond ( $f_{\mathrm{s}}$ ) OPOs up to $1 \mathrm{GHz}$ [14-16]. In addition, for picosecond (ps) OPOs with an extended-cavity, in which the ratio of pump cavity length and the OPO cavity length is a rational number, multi-gigahertz ps signal pulses have been achieved with an $80-\mathrm{MHz}$ pump [17]. As it is the resonating signal that is of interest in such experiments, the cavity requires signal output coupling and the large number of round-trips experienced by the signal pulses before the next pump pulse arrives incurs significant loss. Here we are interested in the non-resonated idler pulses and demonstrate a high-harmonic-cavity OPO in which the resonated signal runs at a repetition frequency as high as 200 times that of the pump without output coupling and for which the corresponding idler pulses have energies up to $1.5 \mu \mathrm{J}$ at $\sim 3 \mu \mathrm{m}$. To the best of our knowledge, these are the highest mid-IR pulse energies reported from a picosecond OPO.

The configuration of the high-harmonic-cavity OPO is shown in Fig. 1. The pump source is a gain-switched diode-seeded Yb-doped-fiber master-oscillator-poweramplifier (MOPA) system employing simple direct amplification, similar to that reported in [18]. It has a central wavelength of $1035 \mathrm{~nm}$ with a pulse duration of $150 \mathrm{ps}$ and a repetition rate of $1 \mathrm{MHz}$. The system delivers $11 \mathrm{~W}$ of average power to the $\mathrm{MgO}$-doped periodically poled $\mathrm{LiNbO}_{3}$ (MgO:PPLN) nonlinear crystal used in these experiments. The power delivered to the crystal was varied using a half-wave plate before a polarizing beam splitter and the orientation of the linear polarization was adjusted by another half-wave plate to realize type-0 $(e+e=e)$ quasi-phase matching. The $40^{-}$ mm-long MgO:PPLN crystal (MOPO1-1.0-40, Covesion)

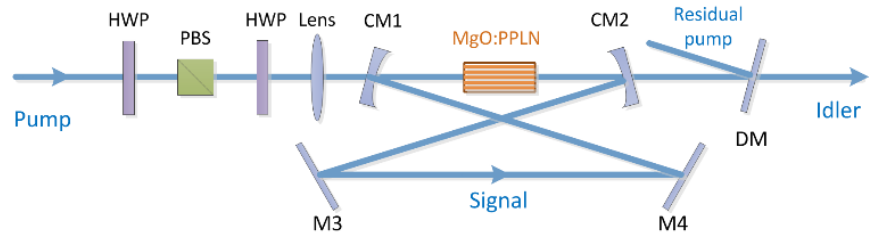

Fig. 1 OPO experimental setup. HWP, half-wave plate; PBS, polarizing beam splitter; CM, curved mirror; DM, dichroic mirror. 
had five gratings with periods ranging from 29.5 to $31.5 \mu \mathrm{m}$ in steps of $0.5 \mu \mathrm{m}$ and was mounted inside an oven to allow temperature tuning in the range from 20 to $200^{\circ} \mathrm{C}$ with a precision of $0.1^{\circ} \mathrm{C}$. The pump beam was focused into the MgO:PPLN crystal with a measured beam waist ( $1 / \mathrm{e}^{2}$ radius of intensity) of $85 \mu \mathrm{m}$. The OPO cavity consisted of a ring cavity comprising two plane mirrors and two concave spherical mirrors (250-mm radius of curvature). The two curved mirrors were set with a separation providing a calculated $90-\mu \mathrm{m}$ beam waist for the signal inside the crystal. All cavity mirrors were coated for high reflectivity (HR) for the signal wavelength range and high transmission for the pump $(90 \%)$ and idler wavelengths (85\%). The cavity length was set to be $1.554 \mathrm{~m}$, corresponding to a fundamental repetition rate of $193 \mathrm{MHz}$ for the OPO, which was the 193rd harmonic of the pump repetition frequency. Each signal pulse therefore makes 193 round-trips in the OPO cavity before temporally coinciding with the next pump pulse. In contrast, the repetition frequency of the idler was the same as that of the pump as it was only generated in the presence of both pump and signal pulses. The idler output is taken through a curved resonator mirror (CM2, Fig. 1) and then a dichroic mirror (DM, Fig. 1) was used to filter out the unconverted pump before the idler power was measured. The idler output power discussed in the following text corresponds to that after mirror CM2, inferred by the losses ( 10\%) of DM.

Figure 2 shows the generated OPO signal pulse train observed as leakage through the HR OPO mirror M3 with a $1 \mathrm{~Gb} / \mathrm{s}$ InGaAs photo detector. The time interval between successive pulses was observed to be $5.18 \mathrm{~ns}$, corresponding to a repetition frequency of $193 \mathrm{MHz}$, as expected. The gradual decrease in signal pulse intensity, until it coincides with the next pump pulse, gives an indication of the round-trip cavity loss. The irregularity in the intensity of several individual pulses is unexplained. To further analyze and understand the intracavity loss that the signal pulses experienced during each roundtrip, we fitted a simple formula $I_{N}=I_{0} \times \alpha^{N}$ to the peak pulse heights of Fig. 2, where $I_{0}$ is the initial pulse intensity transmitted through the cavity mirror, $N$ is the number of

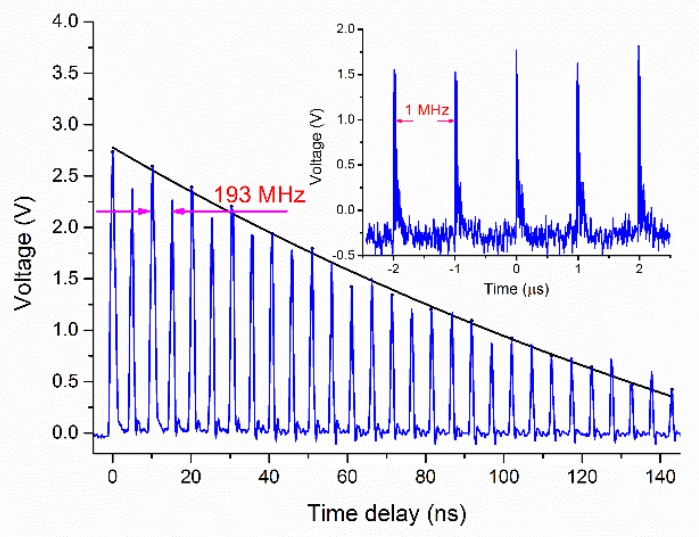

Fig. 2 Pulse trains of signal at $193 \mathrm{MHz}$ repetition frequency, the black curve is fitted by formula in the text. Inset shows the fundamental pulse repetition frequency at $1 \mathrm{MHz}$.

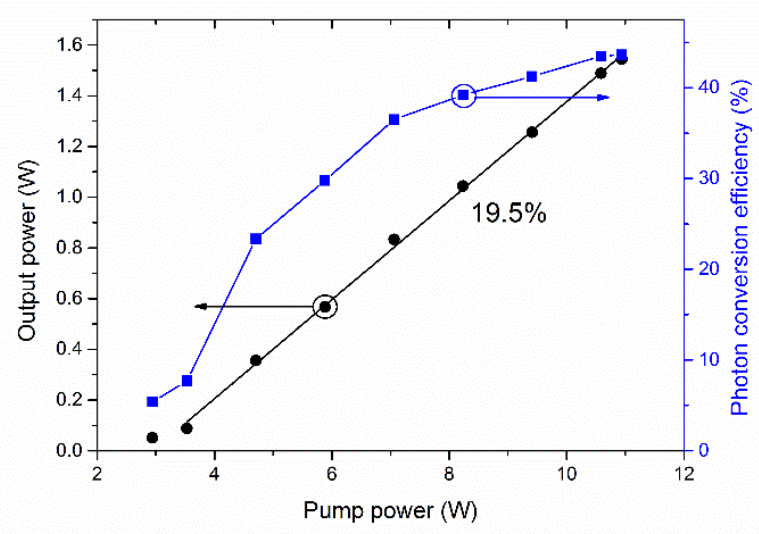

Fig. 3 Idler output power and photon conversion efficiency as a function of pump power. The circles are measured data points, the black solid line is linear fit and the blue solid line is purely to guide the eye.

roundtrips of the signal pulse in the cavity, and $\alpha$ is the round-trip intracavity transmission. A value of 0.98 was obtained, which we attribute mainly to losses from the anti-reflection coatings on the MgO:PPLN crystal and the HR cavity mirrors. As a result, the estimated total intracavity loss after 193 signal round trips, hence the required gain for threshold in the $\mathrm{OPO}$, is $\sim 40 \mathrm{~dB}$. In a recent OPA experiment with the same pump source and crystal, a small-signal gain as high as $79 \mathrm{~dB}$ was observed at pump power levels of $\sim 9 \mathrm{~W}$ [10]. Thus the OPO threshold of $\sim 3 \mathrm{~W}$ observed in this case appears to be in reasonable agreement with our calculated loss and expectations for the gain available from such systems. The inset in Fig. 2 shows the fundamental repetition frequency of $1 \mathrm{MHz}$ when looked at over a longer time scale.

For a fixed PPLN grating with a period of $30 \mu \mathrm{m}$ and a temperature of $90^{\circ} \mathrm{C}$, the $\mathrm{OPO}$ oscillated with a pump threshold of $3 \mathrm{~W}$ and the idler output power increased linearly, with a slope efficiency of $19.5 \%$, reaching a maximum power of $1.5 \mathrm{~W}$, as shown in Fig. 3. The

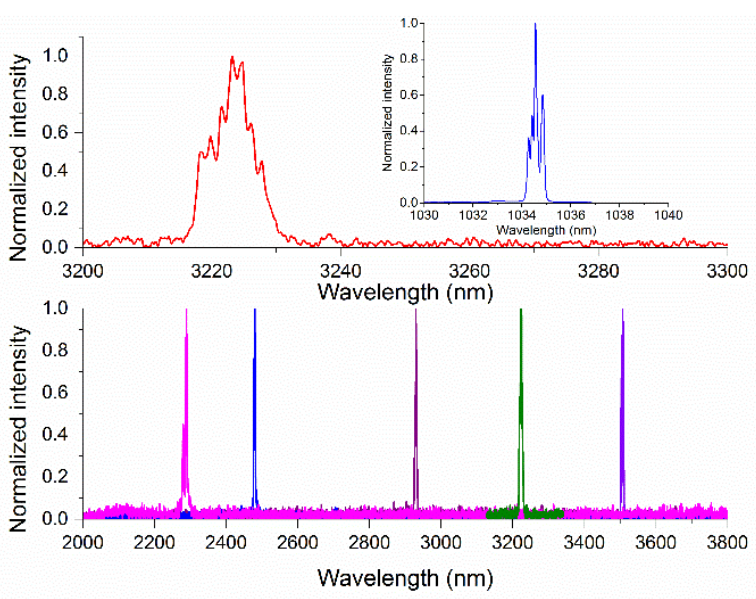

Fig. 4 Idler spectra from the high-harmonically pumped OPO. Fixed wavelength at $3227 \mathrm{~nm}$ (upper), spectrum tuned by using different $\mathrm{MgO}: \mathrm{PPLN}$ gratings and oven temperatures (below). Inset shows the pump spectra. 


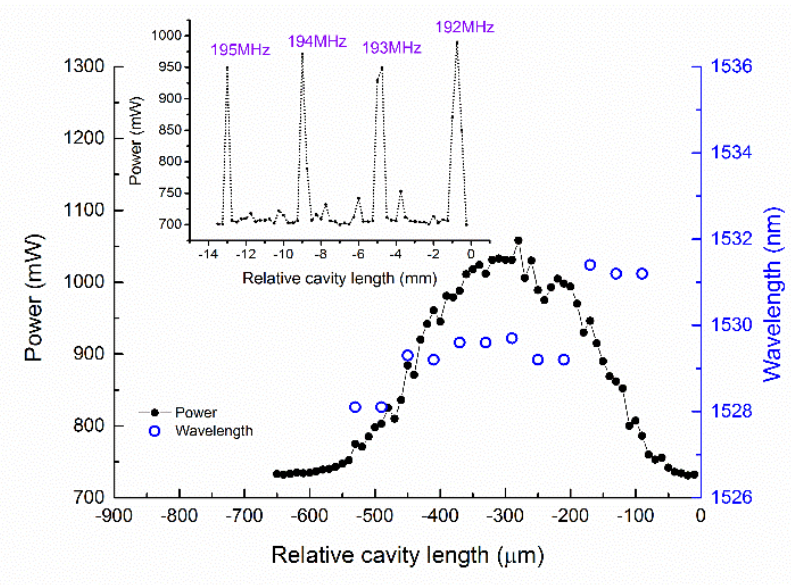

Fig. 5 Variation of idler output power and resonating signal wavelengths with OPO cavity length tuning. Inset shows the output power jumps with larger cavity length tunings at discrete cavity lengths.

corresponding idler pulse energy was therefore $1.5 \mu \mathrm{J}$. The overall external power conversion efficiency at this point was $14 \%$ from pump to the idler, and the photon conversion efficiency corresponded to $43 \%$ with the idler wavelength at $3.2 \mu \mathrm{m}$. It can be seen that the maximum output power was only limited by the available pump power. The idler spectrum was measured using a spectrum analyzer (model 721, Bristol Instruments), showing a central wavelength of $3227 \mathrm{~nm}$ and a full-width at half-maximum (FWHM) spectral bandwidth of $\sim 8 \mathrm{~nm}$. The modulation of the idler spectrum observed in Fig. 4, is attributed to similar modulation on the pump spectrum, shown in inset of Fig. 4, caused by self-phase modulation within the fiber MOPA system. By using different $\mathrm{MgO}$ :PPLN grating periods and oven temperatures, the idler wavelength could be tuned from $2300 \mathrm{~nm}$ to $3500 \mathrm{~nm}$, as given in Fig. 4. All measurements of the spectrum in Fig. 4 are averaged measurement of multiple pulses.

An exploration of the tolerance and influence of the cavity length on the idler output power in the highharmonic-cavity $\mathrm{OPO}$ was performed. As demonstrated in Fig. 5, the idler showed jumps in output power at discrete

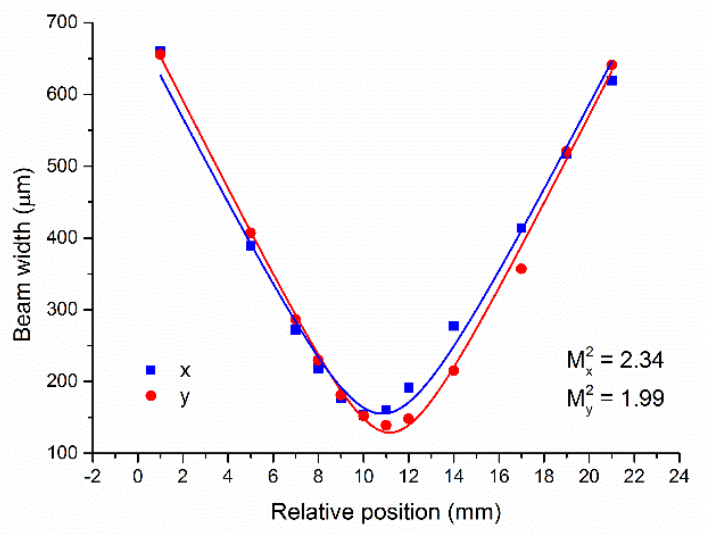

Fig. $6 \mathrm{M}$-squared measurement of idler in horizontal and vertical planes. cavity lengths when tuning over several millimeters. These oscillation peaks corresponded to different harmonic synchronization in the OPO operation spaced at intervals of $\sim 4 \mathrm{~mm}$, in agreement with theoretical expectation. The idler output power did not drop to zero between these peaks as there was some OPG generation when the OPO stopped oscillation. The idler output power versus fine cavity tuning behavior of the OPO, operating at $193 \mathrm{MHz}$ repetition frequency, was then investigated. The total cavity tuning length before the OPO ceased oscillation was $\sim 500 \mu \mathrm{m}$. It is well known that the signal wavelength can be tuned by an adjustment of the cavity length in both ps and fs OPO [19,20]. The cavity-length detuning introduces loss at the signal wavelength by reducing the synchronism between the pump and the phase-matched signal pulses. To maintain synchronism, the signal shifts wavelength within the gain bandwidth to a wavelength with a group velocity that satisfies the new round-trip time to optimize gain. Here we observed that the wavelength of the signal shifted by $\sim 3 \mathrm{~nm}$ when changing the cavity length. The dispersion for signal pulses was accumulated over multiple passes through the $\mathrm{MgO}: \mathrm{PPLN}$ crystal and from reflections on mirrors in the high-harmonic-cavity. The delay of signal pulses with respect to pump pulses becomes smaller with decreasing cavity length, which should result in a shorter signal wavelength when the signal wavelength is in the normal dispersion region of PPLN. This behavior is observed in figure 5. However, the temporal difference due to dispersion of the $40 \mathrm{~mm}$ long PPLN sample between 1528 $\mathrm{nm}$ and $1531 \mathrm{~nm}$ was about $10 \mathrm{fs}$, or less than 2 ps after 193 round trips in the OPO, which is not sufficient to compensate for cavity length detuning. With a FWHM cavity detuning length of 300um for a 193rd-order cavity, a time delay of 193 ps would be generated between the signal and the next pump pulses, which is longer than the 150 ps pump pulse. It may be that such a high-gain system provided a larger tolerance to cavity-length detuning than traditional ps OPOs. It is also possible that a self-adaptation of the resonant beam path occurred in the ring resonator according to a similar process as that investigated in [21] to maintain a constant round-trip time. Without active stabilization or intentionally isolating the OPO from external interference, the OPO still exhibited a good stability with power fluctuation within $\pm 6 \%$ during operation over 30 minutes, mostly due to a power slow drift of the pumping MOPA. The beam quality of the idler was characterized with a Pyrocam-based profiler (NanoScan, Photon). Figure 6 shows the beam propagation factor $\left(M^{2}\right)$ with $M_{x}^{2}=2.34$ and $M_{y}^{2}=1.99$ in the horizontal (parallel to the PPLN zaxis) and vertical planes, respectively, at full power. The difference of $M_{x}^{2}$ and $M_{y}^{2}$ was possibly due to the fact that the pump beam was elliptically distorted in the horizontal plane due to the angle-cleaved end-cap spliced to the output end of the fiber. In addition, the angle-tilted OPO curved mirrors in the horizontal plane could also degrade the $M_{x}^{2}$ due to the astigmatism.

In conclusion, we have demonstrated a compact highenergy, mid-IR, picosecond optical parametric oscillator (SPOPO) employing a high-harmonic-cavity. The OPO is based on a MgO:PPLN crystal and pumped by a nearly 
all-fiber master-oscillator-power-amplifier (MOPA) system. With a cavity that is just a small fraction of the length required to match the pump repetition rate, signal pulses with a repetition rate that is the 193rd harmonic of the $1 \mathrm{MHz}$ pump repetition rate are demonstrated. Pumped by $11 \mu \mathrm{J}, 150 \mathrm{ps}$ pulses at $1035 \mathrm{~nm}$, idler output pulse energies as high as $1.5 \mathrm{\mu J}$ have been achieved, limited only by the available pump energies. The overall photon conversion efficiency reaches $43 \%$ and the idler wavelength can be tuned from $2300 \mathrm{~nm}$ to $3500 \mathrm{~nm}$.

This research was supported by EPSRC Grant $\mathrm{EP} / \mathrm{I02798X/1}$. The data for this paper can be found at 10.5258/SOTON/375602.

\section{References}

1. S. Woutersen, U. Emmerichs, and H. J. Bakker, Science 278, 658-660 (1997).

2. M. R. Papantonakis and R. F. Haglund Jr, Appl Phys A 79, 1687-1694 (2004).

3. V. Z. Kolev, M. W. Duering, B. Luther-Davies, and A. V. Rode, Opt. Express 14, 12302-12309 (2006).

4. D. D. McAlevy Bubb and R. F. Haglund, "Resonant Infrared Pulsed Laser Ablation and Deposition of Thin Polymer Films," in Pulsed Laser Deposition of Thin Films (John Wiley \& Sons, Inc., 2006), pp. 33-61.

5. H. Linnenbank and S. Linden, Opt. Express 22, 18072-18077 (2014).

6. T. V. Andersen, O. Schmidt, C. Bruchmann, J. Limpert, C. Aguergaray, E. Cormier, and A. Tünnermann, Opt. Express 14, 4765-4773 (2006).

7. S. C. Kumar, M. Jelínek, M. Baudisch, K. T. Zawilski, P. G. Schunemann, V. Kubecek, J. Biegert, and M. Ebrahim-Zadeh, Opt. Express 20, 15703-15709 (2012).
8. R. Piccoli, F. Pirzio, A. Agnesi, V. Badikov, D. Badikov, G. Marchev, V. Panyutin, and V. Petrov, Opt. Lett. 39, 4895-4898 (2014).

9. G. Marchev, F. Pirzio, R. Piccoli, A. Agnesi, G. Reali, P. G. Schunemann, K. T. Zawilski, A. Tyazhev, and V. Petrov, Opt. Lett. 38, 3344-3346 (2013).

10. L. Xu, H. Y. Chan, S. U. Alam, D. J. Richardson, and D. P. Shepherd, Opt. Express 23, 12613-12618 (2015).

11. F. Kienle, P. Siong Teh, S. U. Alam, C. B. E. Gawith, D. C. Hanna, D. J. Richardson, and D. P. Shepherd, Opt. Lett. 35, 3580-3582 (2010).

12. T. P. Lamour, L. Kornaszewski, J. H. Sun, and D. T. Reid, Opt. Express 17, 14229-14234 (2009).

13. T. P. Lamour and D. T. Reid, Opt. Express 19, 17557-17562 (2011).

14. D. T. Reid, C. McGowan, W. Sleat, M. Ebrahimzadeh, and W. Sibbett, Opt. Lett. 22, 525-527 (1997).

15. K. A. Ingold, A. Marandi, C. W. Rudy, K. L. Vodopyanov, and R. L. Byer, Opt. Lett. 39, 900-903 (2014).

16. K. Balskus, S. M. Leitch, Z. Zhang, R. A. McCracken, and D. T. Reid, Opt. Express 23, 1283-1288 (2015).

17. O. Kimmelma, S. Chaitanya Kumar, A. Esteban-Martin, and M. Ebrahim-Zadeh, Opt. Lett. 38, 4550-4553 (2013).

18. H. Y. Chan, S. U. Alam, L. Xu, J. Bateman, D. J. Richardson, and D. P. Shepherd, Opt. Express 22, 21938-21943 (2014).

19. M. Ebrahimzadeh, S. French, and A. Miller, J. Opt. Soc. Am. B 12, 2180-2191 (1995).

20. D. C. Edelstein, E. S. Wachman, and C. L. Tang, Appl Phys Lett 54, 1728-1730 (1989).

21. C. Laporte, J. B. Dherbecourt, J. M. Melkonian, M. Raybaut, C. Drag, and A. Godard, J. Opt. Soc. Am. B 31, 1026-1034 (2014). 


\section{References with titles}

1. S. Woutersen, U. Emmerichs, and H. J. Bakker, "Femtosecond Mid-IR Pump-Probe Spectroscopy of Liquid Water: Evidence for a Two-Component Structure," Science 278, 658-660 (1997).

2. M. R. Papantonakis, and R. F. Haglund Jr, "Picosecond pulsed laser deposition at high vibrational excitation density: the case of poly(tetrafluoroethylene)," Appl Phys A 79, 1687-1694 (2004).

3. V. Z. Kolev, M. W. Duering, B. Luther-Davies, and A. V. Rode, "Compact high-power optical source for resonant infrared pulsed laser ablation and deposition of polymer materials," Opt. Express 14, 12302-12309 (2006).

4. D.-D. McAlevy Bubb, and R. F. Haglund, "Resonant Infrared Pulsed Laser Ablation and Deposition of Thin Polymer Films," in Pulsed Laser Deposition of Thin Films(John Wiley \& Sons, Inc., 2006), pp. 33-61.

5. H. Linnenbank, and S. Linden, "High repetition rate femtosecond double pass optical parametric generator with more than $2 \mathrm{~W}$ tunable output in the NIR," Opt. Express 22, 18072-18077 (2014).

6. T. V. Andersen, O. Schmidt, C. Bruchmann, J. Limpert, C. Aguergaray, E. Cormier, and A. Tünnermann, "High repetition rate tunable femtosecond pulses and broadband amplification from fiber laser pumped parametric amplifier," Opt. Express 14, 4765-4773 (2006).

7. S. C. Kumar, M. Jelínek, M. Baudisch, K. T. Zawilski, P. G. Schunemann, V. Kubecek, J. Biegert, and M. EbrahimZadeh, "Tunable, high-energy, mid-infrared, picosecond optical parametric generator based on $\mathrm{CdSiP}_{2}$," Opt. Express 20, 15703-15709 (2012).

8. R. Piccoli, F. Pirzio, A. Agnesi, V. Badikov, D. Badikov, G. Marchev, V. Panyutin, and V. Petrov, "Narrow bandwidth, picosecond, $1064 \mathrm{~nm}$ pumped optical parametric generator for the mid-IR based on $\mathrm{HgGa}_{2} \mathrm{~S}_{4}$," Opt. Lett. 39, 4895-4898 (2014).

9. G. Marchev, F. Pirzio, R. Piccoli, A. Agnesi, G. Reali, P. G. Schunemann, K. T. Zawilski, A. Tyazhev, and V. Petrov, "Narrow-bandwidth, 100 ps seeded optical parametric generation in $\mathrm{CdSiP}_{2}$ pumped by Raman-shifted pulses at 1198 nm," Opt. Lett. 38, 3344-3346 (2013).

10. L. Xu, H. Y. Chan, S. U. Alam, D. J. Richardson, and D. P. Shepherd, "High-energy, near- and mid-IR picosecond pulses generated by a fiber-MOPA-pumped optical parametric generator and amplifier," Opt. Express 23, 12613-12618 (2015).

11. F. Kienle, P. Siong Teh, S.-U. Alam, C. B. E. Gawith, D. C. Hanna, D. J. Richardson, and D. P. Shepherd, "Compact, high-pulse-energy, picosecond optical parametric oscillator," Opt. Lett. 35, 3580-3582 (2010).

12. T. P. Lamour, L. Kornaszewski, J. H. Sun, and D. T. Reid, "Yb:fiber-laser-pumped high-energy picosecond optical parametric oscillator," Opt. Express 17, 14229-14234 (2009).

13. T. P. Lamour, and D. T. Reid, "650-nJ pulses from a cavitydumped Yb:fiber-pumped ultrafast optical parametric oscillator," Opt. Express 19, 17557-17562 (2011).

14. D. T. Reid, C. McGowan, W. Sleat, M. Ebrahimzadeh, and W. Sibbett, "Compact, efficient $344-\mathrm{MHz}$ repetition-rate femtosecond optical parametric oscillator," Opt. Lett. 22, 525527 (1997).

15. K. A. Ingold, A. Marandi, C. W. Rudy, K. L. Vodopyanov, and R. L. Byer, "Fractional-length sync-pumped degenerate optical parametric oscillator for $500-\mathrm{MHz} 3-\mu \mathrm{m}$ mid-infrared frequency comb generation," Opt. Lett. 39, 900-903 (2014).

16. K. Balskus, S. M. Leitch, Z. Zhang, R. A. McCracken, and D. T. Reid, "1-GHz harmonically pumped femtosecond optical parametric oscillator frequency comb," Opt. Express 23, 12831288 (2015).

17. O. Kimmelma, S. Chaitanya Kumar, A. Esteban-Martin, and M. Ebrahim-Zadeh, "Multi-gigahertz picosecond optical parametric oscillator pumped by $80-\mathrm{MHz}$ Yb-fiber laser," Opt. Lett. 38, 4550-4553 (2013).

18. H. Y. Chan, S. U. Alam, L. Xu, J. Bateman, D. J. Richardson, and D. P. Shepherd, "Compact, high-pulse-energy, highpower, picosecond master oscillator power amplifier," Opt. Express 22, 21938-21943 (2014).

19. M. Ebrahimzadeh, S. French, and A. Miller, "Design and performance of a singly resonant picosecond $\mathrm{LiB}_{3} \mathrm{O}_{5}$ optical parametric oscillator synchronously pumped by a self-modelocked Ti:sapphire laser," J. Opt. Soc. Am. B 12, 2180-2191 (1995).

20. D. C. Edelstein, E. S. Wachman, and C. L. Tang, "Broadly tunable high repetition rate femtosecond optical parametric oscillator," Appl Phys Lett 54, 1728-1730 (1989).

21. C. Laporte, J. B. Dherbecourt, J. M. Melkonian, M. Raybaut, C. Drag, and A. Godard, "Analysis of cavity-length detuning in diffraction-grating narrowed picosecond optical parametric oscillators," J. Opt. Soc. Am. B 31, 1026-1034 (2014). 